\title{
Determination of analytical precision in calculating total energy expenditure using the doubly labelled water method in humans
}

\author{
R. Baker, K. Donkers, E.R. Orford, P.A. Singh and M.C. Venables \\ Stable Isotope Facility, MRC Elsie Widdowson Laboratory, 120 Fulbourn Road, Cambridge, CB1 9NL, UK
}

Doubly labelled water (DLW) is the gold standard method for estimation of free-living energy expenditure in humans ${ }^{(1)}$. Overall precision is an important factor to consider when designing a study, as poor precision in total energy expenditure (TEE) will increase the sample size required. The precision or analytical variation of modelled TEE is variable and dependent on the laboratory, instrumentation used and specific methodology used. In order to calculate the precision of our methodology, we assessed the reproducibility of modelled TEE estimation over ten consecutive weeks. Additionally, jack-knife resampling was used to assess bias within an individual estimate of TEE.

Two female participants (mean (SD): age, 40 (4) years: height, $1.66(0.04) \mathrm{m}$ : weight, $77.6(9.1) \mathrm{kg}$ ) were dosed with $150 \mathrm{mg} / \mathrm{kg}$ body weight $\mathrm{H}_{2}^{18} \mathrm{O}$ (Taiyo Nippon Sanso, Tokyo, Japan) and $80 \mathrm{mg} / \mathrm{kg}$ body weight ${ }^{2} \mathrm{H}_{2} \mathrm{O}$ (Cambridge Isotope Laboratories, Massachusetts, USA). The protocol was approved by the East of England - Cambridge Central Research Ethics Committee, Cambridge, UK. Eleven urine samples were collected, comprising of a baseline urine sample followed by ten consecutive days postdosing, avoiding the first void of the day. To minimise freeze-thaw cycles, each urine sample was aliquoted into ten $7 \mathrm{ml}$ glass bijou bottles and stored at $-20^{\circ} \mathrm{C}$ until analysis. Analysis of $\mathrm{H}_{2}^{18} \mathrm{O}$ and ${ }^{2} \mathrm{H}_{2} \mathrm{O}$ was conducted weekly using a continuous-flow isotope ratio mass spectrometer (AP2003, Analytical Precision Ltd., Northwich, Cheshire, UK) and a dual-inlet isotope ratio mass spectrometer (IsoPrime, GV Instruments Ltd., Wythenshawe, Manchester, UK) respectively. Energy expenditure was modelled according to Schoeller ${ }^{(2)}$ and expressed in kilojoules. Precision was calculated as the coefficient of variation.

Average daily TEE for repeat measurements was $8643(52) \mathrm{kJ}$ and $8683(52) \mathrm{kJ}$ (mean (SE)) for participant Y \& W respectively. Both had total analytical precisions of $1.20 \%$, with separate precisions for isotope elimination rates and dilution spaces reported in Table 1 . Jack-knife resampling confirmed a standard error of the TEE estimate of 63 and $127 \mathrm{~kJ}$ and a small mean bias of 12 and $-15 \mathrm{~kJ}$.

Table 1. Isotope elimination rates and dilution spaces for the two participants.

\begin{tabular}{|c|c|c|c|c|c|c|c|c|c|c|}
\hline & \multicolumn{2}{|c|}{$\mathrm{K}_{\mathrm{o}}$} & \multicolumn{2}{|c|}{$\mathrm{K}_{\mathrm{h}}$} & \multicolumn{2}{|c|}{$\mathrm{N}_{\mathrm{o}}$} & \multicolumn{2}{|c|}{$\mathrm{N}_{\mathrm{h}}$} & \multicolumn{2}{|c|}{ TEE } \\
\hline & $\mathrm{d}^{-1}$ & $\% \mathrm{CV}$ & $\mathrm{d}^{-1}$ & $\% \mathrm{CV}$ & $\mathrm{kg}$ & $\% \mathrm{CV}$ & $\mathrm{kg}$ & $\% \mathrm{CV}$ & $\mathrm{kJ}$ & $\% \mathrm{CV}$ \\
\hline Participant Y & 0.0970 & 0.13 & 0.0742 & 0.27 & 31.06 & 0.06 & 31.98 & 0.06 & 8643 & 1.20 \\
\hline Participant W & 0.1058 & 0.24 & 0.0851 & 0.14 & 35.12 & 0.19 & 36.16 & 0.19 & 8683 & 1.20 \\
\hline
\end{tabular}

Preliminary data for four repeat measurements. Values are means with $\% \mathrm{CV}$.

$\mathrm{k}_{\mathrm{o}}$, oxygen elimination rate; $\mathrm{k}_{\mathrm{h}}$, hydrogen elimination rate; $\mathrm{N}_{\mathrm{o}}$, oxygen dilution space; $\mathrm{Nh}$, hydrogen dilution space; TEE, total energy expenditure.

In conclusion, the analytical precision for our laboratory when using the doubly labelled water method to calculate TEE is well within the published limits of 3-6\% $\%^{(3,4)}$.

1. Schoeller DA \& van Santen E. (1982) J Appl Physiol Respir Environ Exerc Physiol 53(4), 955-9.

2. Schoeller DA, Ravussin E, Schutz Y et al. (1986) Am J Physiol 250(5 Pt 2), R823-30.

3. Schoeller DA, Taylor PB \& Shay K (1995) Obesity Research 3(1), 15-20.

4. Cole TJ \& Coward WA (1992) Am J Physiol 263(26), E965-E973. 